\title{
Algunas nociones y aplicaciones de la investigación documental denominada estado del arte
}

\author{
Nubia Leonor Posada González*
}

Artículo recibido:

16 de enero de 2015

Artículo aceptado:

13 de octubre de 2016

\section{Resumen}

En este artículo se actualizan las direcciones de los referentes que aún permanecen en la Web y que fueron trabajados en la tesis doctoral en filosofía Carlos Cardona: Estado del Arte, defendida por la autora dentro del programa "La complejidad de la razón" del Departamento de Filosofía de la Universidad de Málaga, España, en 2011.

Se ha hecho el rastreo, detección, obtención, consulta, extracción, recopilación de información y análisis especulativo de algunas definiciones de estado del arte, obteniendo como producto otras dos, que se aplican a la obra del filósofo de Tiana, Carlos Cardona Pescador, y lo producido sobre él, en lo que se le cita o menciona.

* Universidad de la Sabana. Sede Puente del Común, Colombia

nubia.posada@unisabana.edu.co

INVESTIGACIÓN BIBLIOTECOLÓGICA, Vol.31, Núm.73, septiembre/diciembre, 2017, México, ISSN: 2448-8321. pp. 237-263 
El artículo corresponde a la segunda parte de la investigación; se ha tenido en cuenta el modo como el término estado del arte se refleja en el trabajo intelectual de la institución académica y de algunas organizaciones internacionales, y se ha concluido la conveniencia de un tratamiento más sistemático en la conceptualización del estado del arte como una modalidad investigativa que tiene impacto en todos los saberes científicos que contribuyen al desarrollo humano.

Palabras clave: Documentación; Investigación Documental; Teoría de Investigación; Estado del Arte; Definición; Carlos Cardona Pescador; Filosofía Actual.

\title{
Some ideas and applications of "State of the Art" documental research \\ Nubia Leonor Posada-González
}

\begin{abstract}
This paper serves to update the addresses web pages still available on the web which were in the doctoral thesis in philosophy Carlos Cardona: State Estado del arte, defended by the author within the program "The complexity of reason" offered by the Faculty of Philosophy of the University of Malaga, Spain, in 2011. Tracking, detecting consulting, summarizing, and speculative analysis of the definition of the State of the Art was performed, which yielded two additional definitions used in the work of the philosopher Carlos Cardona Pescador, or in works that cite or mention him. This article belongs to the second part of the research. Researchers have taken into account that the expression "State of the Art" and the way it has permeated the intellectual work of the academic institution and international organizations, concluding that a systemic conceptualization of the idea of state-of-the-art research that impacts scientific knowledge that contributes human development would be useful.
\end{abstract}

Keywords: Documentation; Documental Research; Research Theory; State of the Art; Meaning; Carlos Cardona Pescador; Current Philosophy. 


\section{INTRODUCCIÓN}

T a presente investigación tuvo como fin realizar el primer estado del arte Lsobre Carlos Cardona Pescador, su obra y los difusores de la misma. Para lograrlo se estructuraron dos definiciones de esta modalidad investigativa, después de un análisis teórico y metodológico que es el tema de este artículo y que se presenta en su segunda parte. Aplicando las nuevas definiciones, se compiló la obra intelectual de y sobre Carlos Cardona según su cobertura en España y en el contexto internacional, esclareciendo algunos subtemas relevantes para entender la vida, obra y aportes originales de este autor. Se elaboraron referentes para la construcción del perfil crítico documental desde las categorías "bio-bibliografía”, "riqueza temática” y "algunas huellas de su modo de construir productos intelectuales". Teseo presenta así esta pesquisa:

Investigación sobre la obra filosófica de Carlos Cardona Pescador. Se divide en una introducción, en la que se articula una investigación biobibliográfica de Carlos Cardona. Se enfatiza la vinculación del trabajo intelectual, estudios, referentes de su pensamiento, vínculos, principales productos intelectuales y perfiles con que se le publicita, corrigiendo y complementando lo compilado, con algunos datos obtenidos en este trabajo. Se parte así del planteamiento cardoniano de recuperación de la unidad. Ante las preguntas acerca de cómo y por qué conocer científicamente al autor, su obra y lo producido con ocasión de él: se plantea la conveniencia de elaborar un Estado del Arte, sobre Carlos Cardona y los estudiosos de su obra. Para ello, la autora recurre a una amplia discusión sobre la teoría y el método del Estado del Arte, en la que se proponen dos definiciones del mismo. A continuación la autora pasa a elaborar un perfil crítico documental de Carlos Cardona desde las categorías "biobibliografía", "riqueza temática" y "algunas huellas de su modo de construir productos intelectuales. (Gobierno de España, s. f.)

La metodología es la propia de esta modalidad de investigación acerca de la obra de y sobre un autor, escuela o tema, o de pesquisas sobre un asunto específico. Su enfoque integra características tanto de investigación cualitativa como cuantitativa; es una investigación básica en el sentido de producir, a partir de un análisis teórico especulativo-trascendiendo la actividad revisionista y repetitiva-dos propuestas de nuevas definiciones de la modalidad investigativa denominada estado del arte. Es documental y a la vez aplicada porque resuelve el problema práctico de la necesidad de compilar, en las circunstancias posibles, la producción intelectual de un autor, como requisito previo para cualquier otra investigación científica o línea de investigación rigurosa sobre él (Hernández, Fernández y Baptista, 2010). Es además bibliográfica, descriptiva, conceptual, sistemática, hermenéutica, de construcción 
interdisciplinaria y de producción continua, por las necesarias sucesivas actualizaciones (Hernández, Fernández y Baptista, 2010: 487-488, 600).

En este artículo se parte de lo que se entiende por estado del arte en el contexto local, para luego extenderse a nivel nacional e internacional, avanzando en subtemas de mayor complejidad de análisis del término. Se comienza por su comprensión en el trabajo académico de la Universidad de Málaga, se avanza hasta llegar a las dos nuevas definiciones y a una síntesis sobre los aportes del estado del arte como una modalidad de investigación que garantiza la calidad de la investigación científica en el modo de recoger lo que se sabe acerca de lo que se quiere profundizar y facilita la máxima aproximación a la universalidad de fuentes y un perfil crítico documental.

A causa del tratamiento hermenéutico, el texto es necesariamente abundante en citas y análisis crítico de todos los subtemas de cada definición comparada, como medio para consolidar dos definiciones filosóficamente más consistentes que las compiladas. Por la complejidad y abundancia del trabajo analítico, se van obteniendo conclusiones parciales al desglosar los subtemas, las cuales se sintetizan al final del artículo.

\section{El término eStAdo DEL ARTE EN EL TRABAJO ACADÉMICO DE LA Universidad de Málaga}

En esta etapa de la pesquisa (Posada, 2011: 93-131) el motor de búsqueda Google arrojó los siguientes resultados con los términos "Universidad de Málaga Estado del Arte”: sin que conste la fecha y como frutos pedagógicos, se describe “[...] el curso objeto de análisis ha evolucionado de manera gradual en los últimos años, sin variaciones traumáticas en cuanto a impartición. [...] Como ejemplo, podemos mencionar algunos de estos trabajos: [...] Estado del arte en Localización y Algoritmos Evolutivos [...] Estado del Arte en Diseño Evolutivo" (Alba y Cotta, s. f.: 4-5). La información fechada más antigua es de 2004, cuando dos profesores del grupo DIANA del Plan Andaluz de Investigación ofrecieron el curso Realidad virtual y presencia, cuyo objetivo era "[...] dar una visión global del Estado del Arte de la realidad virtual y plantear los retos a los que se enfrentan los investigadores para hacer de esta tecnología una herramienta de uso común” (Diaz y Reyes, 2004: 1). Entre los contenidos se lee:

Tras una introducción histórica, se presenta una primera aproximación a la realidad virtual basada en la estimulación de los sentidos, con especial atención a la vista, el oído y el tacto. Para ello, primero se introducen los fundamentos fisiológicos 
de estos sistemas sensoriales. A continuación se analizan los periféricos empleados para excitar dichos sistemas, presentando el Estado del Arte de la tecnología. (Diaz y Reyes, 2004: 1)

Al año siguiente, el Departamento de Fruticultura Subtropical y Fitopatología ofreció el curso doctoral "Frutales tropicales: biotecnología del chirimoyo: Estado del Arte” (Universidad de Vigo, 2005: 3). En 2006 la Universidad de Málaga se convirtió en la quinta en España que entró en el Programa Campus de Inter Systems, en el que "[...] las instituciones docentes desarrollan proyectos educativos y acceden al Estado del Arte en los campos del desarrollo rápido de aplicaciones” (Universidad de Málaga, 2006: 1).

En abril de 2008 se realizó la Jornada Universidad-Empresa para “[...] ofrecer una visión del Estado del Arte y de las líneas de investigación en las que trabajan dos destacados grupos de investigación de la UMA, en dos sectores más destacados en los últimos años: tecnologías inalámbricas y sector energético" (Universidad de Málaga, 2008). En el mismo año se leía en la Carta de Servicios de la Oficina de Transferencia de Resultados de Investigación (OTRI):

En relación con la Protección Industrial e Intelectual: [...] 3.3. Recopilación y análisis del "Estado del Arte" en 5 días, en el 80\% de los casos. [...] Con el fin de verificar el nivel obtenido en el cumplimiento de los compromisos adquiridos en esta Carta por la OTRI de la Universidad de Málaga, se establecen los siguientes indicadores del nivel de calidad y eficacia de los servicios prestados por esta oficina: [...] 3. Respecto a la Protección Industrial e Intelectual: 3.3. Porcentaje de recopilaciones y análisis del «Estado del Arte» realizados en 5 días. (Universidad de Granada, 2009).

En julio de 2009 participó la Universidad de Málaga, con la intervención "Realidad Aumentada en dispositivos móviles. Tecnología y Estado del Arte", en el Curso Animación digital y realidad aumentada (Universidad de Granada, 2009).

De lo anterior se concluye que, por lo menos desde 2004, en la Universidad de Málaga se socializa el trabajo que tiene en cuenta el estado del arte a nivel de profesores y en el alumnado de primero y tercer nivel -doctorado-, aunque las instancias parecen no generalizadas, siendo el grupo de informática el más fuerte en esta modalidad. Sólo consta la producción de trabajo de dos alumnos sobre el estado del arte. En filosofía no se ha hallado información relacionada con el tema. Las fuentes consultadas no han arrojado datos suficientes para discernir si realmente se está denominando estado del arte a la elaboración, dentro de la misma Universidad, de investigaciones científicas que correspondan a esta modalidad de investigación documental. 


\section{Algunos ejemplos del término estado del arte en EL trabajo FILOSÓFICO EN ESPAÑOL}

A 31 de mayo de 2009, utilizando los términos "filosofía Estado del Arte", el motor de búsqueda Google, ha arrojado ocho resultados:

España

1. Reporte de un capítulo publicado en Brasil en la memoria del año 2002 del Departamento de Filosofía de la Universidad de Oviedo: "Ciência, Tecnologia e Sociedade: O estado da arte na Europa e nos Estados Unidos" (Universidad de Oviedo, 2002: 393). ${ }^{1}$

\section{México}

2. En el seminario "El estado del arte en el ámbito educativo del CAMDF", el relato de una opinión es más específico de otro tema:

[...] en lo correspondiente a los trabajos de la segunda parte, referidos a la educación basada en normas de competencia didáctica, quienes integramos el Área de Filosofía nos pronunciamos por un abierto rechazo a sus presupuestos teóricos fundamentales que sin duda son contrarios a la orientación filosófica que postula el artículo $3^{\circ}$ constitucional. (Vargas, 2009: 26)

\section{Colombia}

3. Seminario taller Condiciones de posibilidad de la filosofía y su enseñanza:

Temáticamente, el seminario-taller comparte con los participantes el estado del arte o Status Quaestionis de los dos temas que enlaza -la filosofía y su enseñanza[sic]. Pone en manos de los estudiantes del primer año de la Licenciatura en Filosofía de la Universidad Pedagógica Nacional tanto materiales como cuestiones de frontera (cutting edges) que pueden ser profundizados tanto en el desarrollo de los estudios como en los futuros trabajos de grado. (Universidad Pedagógica Nacional, 2009: 2)

4. Un programa de Maestría en Filosofía: "La metodología de trabajo será la siguiente: durante la primera parte del seminario (7 u 8 sesiones) el profesor presenta el estado del arte y el campo problemático a trabajar". (Universidad del Rosario, s. f.). 
5. Una publicación: "Cabe destacar que en su exposición del Estado del Arte, Abellán considera que el problema no ha avanzado mucho desde que Menéndez Pelayo lo formuló en la polémica sobre la ciencia española”. (Torregoza, 2006: 359).

6. Un libro: "Duav, G., Los tres órdenes a lo imaginario del feudalismo, Argot, Barcelona, 1983. Para el Estado del Arte contemporáneo sobre la Edad Media y el Feudalismo remitimos a Guerreau, A., Féodalité. En: Dictionaire Raissoné de l' Occident Médiéval. Op. cit., pp. 387-406. Contiene una extensa e intensa bibliografía en pp. 595- 609”, pie de página p. 138, y "Para un Estado del Arte y de la cuestión de Siger y del Averrísmo [sic], véase: Sanga1li, I. "Consideracoes sobre a historiografía do <averroísmo>”. En Veritas, 49 (2004) 489-505”, pie de página 868 (Soto, 2007: 44, 256).

7. Una revista de filosofía de la ciencia: respecto a la contextualización, en la primera parte de los artículos que reportan resultados de investigaciones científicas: "[...] es una de las columnas vertebrales del método científico que contribuye a identificar y sustentar el problema a estudiar y su contexto dentro del Estado del Arte, y que sirve además de elemento de análisis para el correspondiente juicio de pertinencia por cuenta de la comunidad científica”. (Monroy, 2004: 46)

8. Un subtema de proyecto de tesis doctoral: Estudios sobre la relación entre Schiller y Hölderlin: Estado del Arte.

El Estado del Arte que puedo presentar en este proyecto es el resultado de la pesquisa bibliográfica que he llevado a cabo en el último año. Aunque existe una enorme cantidad de bibliografía acerca de cada uno de los autores por separado, como señalaré más adelante, ha sido curioso para mí encontrar que son muy pocos los estudios que se dedican a comparar el pensamiento filosófico de Schiller y Hölderlin. He tenido acceso, entre otros, al Philosopher's Index, a la bibliografía internacional de Hölderlin en Internet y a la biblioteca del congreso en Washington (donde se almacenan todos los títulos publicados en inglés); además de la bibliografía mencionada por todos los autores consultados hasta el momento. Son muy pocos los libros que he encontrado que se dediquen a relacionar la producción de ambos autores.

[...] No he podido acceder a la lista completa ni del Hölderlin Jahrbuch, editado por la Hölderlin Gesellschaft en Tubinga, ni del Schiller Jahrbuch, editado por la Deutsche Schiller Gesellschaft, por lo que es probable que existan algunos artículos sobre el tema, que no me han sido hasta ahora accesibles. (Acosta, 2005: 1-2) 
Esta indagación ejemplifica que la incursión del estado del arte en cuanto modalidad de investigación teórica no es deducible del modo como se menciona el término en las primeras publicaciones y actividades académicas, pero las dos últimas sí facilitan elementos de reflexión.

En 2004 Sonia Esperanza Monroy resaltó la relación directa entre el estado del arte y el juicio de pertinencia de la comunidad científica, al afirmar en una revista sobre filosofía de la ciencia que "[...] es una de las columnas vertebrales del método científico que contribuye a identificar y sustentar el problema a estudiar y su contexto dentro del Estado del Arte, y que sirve además de elemento de análisis para el correspondiente juicio de pertinencia por cuenta la comunidad científica" (Monroy, 2004: 46).

En 2005 María del Rosario Acosta, en una propuesta anterior a la definitiva de su tesis doctoral en filosofía, hace notar que tiene conciencia de la diferencia entre un estado del arte como modalidad investigativa documental, en el que se intenta hacer un rastreo lo más completo posible, y una etapa compilatoria de una investigación en la que se hace un avance importante pero reconociendo que falta información para que pueda llamarse con propiedad estado del arte. La doctoranda tiene la claridad de especificar que le puede faltar información de fuentes relevantes.

\section{EL TÉRMINO ESTADO DEL ARTE EN DOS EJEMPLOS DE DIAGNÓSTICO EDUCATIVO PARA GENERAR NUEVOS DIRECCIONAMIENTOS DE LA CULTURA HISPANOAMERICANA}

En 1995 culminó el estudio Reformas educativas comparadas. Bolivia, México, Chile, España. Estado del Arte del CEBIAE (La Paz, Bolivia) que, según el resumen publicado por la Unesco, consistió en una

Investigación documental que analiza y contrasta los principales planteamientos de las leyes educativas de México, España [sic] Chile y Bolivia, con el propósito de aportar a las instituciones con elementos de reflexión y discusión sobre las orientaciones existentes en las reformas de los indica dos países. Expone la necesidad de generar y movilizar políticas de apoyo y concertar acciones tendientes a resolver necesidades primarias de la población escolar. Destaca que "[...] los conocimientos básicos constituyen la base para edificar otros niveles de educación". (Unesco, 2005: 266)

Acerca de la investigación más antigua, en español, del estado del arte localizada en la Web durante este estudio, se halla un resumen en la base de datos de la Red Latinoamericana de Información y Documentación en Educación (REDUC); en este lugar se reportan los resúmenes de otras 29 investi- 
gaciones sobre estado del arte (Corvalán, 1985). ${ }^{2}$ Posteriormente, el Centro para la Investigación y la Innovación Educativas [Centre for Educational Research and Innovation, CERI] de la Organización para la Cooperación y el Desarrollo Económico (OCDE), publicó la investigación Políticas educativas sobre nuevas tecnologías en los países iberoamericanos, que tenía

[...] como objetivo primordial ofrecer una visión panorámica del Estado del Arte en el ámbito de las relaciones entre las nuevas tecnologías de la información y de la comunicación (TIC) y la educación escolar, prestando especial atención a su vinculación con los procesos de innovación educativa y sus implicaciones, tanto políticas como institucionales y, por supuesto, pedagógicas, en los países iberoamericanos. (Benavides y Pedró, 2007: 19)

El TÉRMINO ESTADO DEL ARTE EN UN EJEMPLO DE UNIVERSIDAD ESPAÑOLA QUE LO EXIGE EN EL PROCESO DE SEGUIMIENTO Y EVALUACIÓN DE TESIS DOCTORALES

En una universidad madrileña se lee:

Durante la Fase 2 el doctorando bajo la supervisión del director y apoyo del tutor irá analizando el marco conceptual o las teorías en las que se pueda y desee enmarcar la Tesis, concretando el "Estado del Arte" en la construcción inicial de la propuesta de un modelo de análisis explicativo de los objetivos pretendidos y que facilite el buen fin de la Tesis como respuesta buscada a los interrogantes planteados [...] En la Segunda Reunión se abordarán las cuestiones siguientes: Presentación sintética por el doctorando del "Estado del Arte" o de referencia teórica de la Tesis. (Universidad Autónoma de Madrid, s. f.: 4)

Por la redacción del texto parecería que faltara diferenciar entre la inclusión de la información aportada por investigaciones de estado del arte durante la etapa compilatoria y la concreción de las distintas fuentes documentales que se eligen como sustento de la investigación sobre un tema específico.

2 Compila y analiza 149 estudios sobre el tema. Toma también datos de CPES, Paraguay. Analiza fundamentalmente información disponible en el REDUC. 


\section{El TÉRMINO ESTAdo DEL ARTE EN UN EJEMPLO ESPAÑOL DE RECURSO UNIVERSAL DE INVESTIGACIÓN CIENTÍFICA}

En octubre de 2004 la empresa CINDOC-CSIC culminó la investigación Revistas cientificas electrónicas: Estado del Arte, facilitando a la academia y a la comunidad científica internacional un referente para la creación y puesta al día en la calidad y acceso de este tipo de publicaciones.

Algunos de los temas del estado del arte hacen referencia a las definiciones, el contexto histórico, características del objeto de estudio, su novedad, cantidad, diversas clasificaciones, la relación entre el lugar en el que realiza la investigación y el contexto universal deducido de la investigación misma, tendencias y visión prospectiva, características relacionadas con la producción del material objeto de estudio, sus diferentes lenguajes y las causas y relaciones de los mismos, contexto, lugares y etapas de producción, y particularidades de su difusión.

También aporta alternativas de solución al problema de la obsolescencia tecnológica: "Este hecho se está produciendo de forma continua tanto en hardware como en software. Por tanto, debe preverse a través de diferentes medidas y garantizar la conservación, acceso, lectura e integridad de los documentos" (CINDOC-CSIC, 2004: 38).

Incluye información sobre los responsables de la conservación del material objeto de estudio, la integridad y autenticidad de sus contenidos, el posicionamiento de material objeto de estudio en el contexto local, internacional y mundial, los derechos de autor, una clasificación de la producción intelectual, las clases de derecho que se tiene sobre producción intelectual y el modo como son protegidas por la ley, y bibliografía.

El trabajo también expone los problemas relacionados con el monopolio económico que impide universalizar rápidamente los avances científicos al encarecer el acceso a la información:

Para situarnos en el mundo de las cifras que se barajan en este ámbito, el valor medio de una revista oscila en unos $1.500 €$. Actualmente se publican unas 24.000 revistas que sacan a la luz unos 2.500.000 artículos al año. En definitiva, hablamos de un negocio de unos 10.000 millones de $€$ /año, con un margen de beneficio aproximado de un $30 \%$. Ante esta situación, hasta las organizaciones ricas como el caso de la Universidad de California que paga en subscriciones [sic] 30 millones de $€$ tiene problemas para seguir afrontando estos costes. (CINDOC-CSIC, 2004: 112) ${ }^{3}$

3 Entre los antecedentes se lee: "Algo muy distintivo, y por cierto no muy frecuente, es lo que sucedió en Dinamarca en 1998 cuando el gobierno nombró una comisión de expertos educativos de Noruega y del Reino Unido para que realizaran una evaluación del Estado del Arte en materia de tecnologías escolares antes de proceder al diseño de su plan político". (Benavides y Pedró, 2007: 57) 


\section{El ESTADO DEL ARTE, MODALIDAD INVESTIGATIVA}

Según Lizcano, "El Estado del Arte es ilustrativo, focaliza y sintetiza un campo del conocimiento, en este sentido trata de hacer una enseñanza práctica de las circunstancias en que se desarrolla" (Universidad de la Sabana, s. f.). La noción de "campo del conocimiento" es tan amplia que podría incluirse en cualquier unidad de producción intelectual que presente alguna novedad, incluyendo una investigación sobre meta-teorías. Esto parece evidenciar la necesidad de buscar otros referentes para esclarecer el término estado del arte.

La enseñanza práctica suele ser fruto de un esfuerzo teórico que ha surgido de lo real; el estado del arte es frecuentemente reconocido como una modalidad de investigación acerca de la investigación (Ramírez, 2006: 12) o sobre el conocimiento producido (Rodríguez y Acosta, 2007: 39), una investigación teórica (Rodríguez y Acosta, 2007: 38) documental (Rodríguez y Acosta, 2007: 38; García, s. f.: 2; Manrique y Zapata, 2006: 7; Souza, s. f.: 1) y compilatoria (Escuela Latinoamericana de Cooperación y Desarrollo, 2005: 8) pero también con diseño de campo (Leal, 2006: 2; Diez, 2003: 1), ${ }^{4}$ bibliográfica (Manrique y Zapata, 2006: 21; Rodríguez y Acosta, 2007: 38), descriptiva (Ramírez, 2006: 13; Rodríguez y Acosta, 2007: 39; Leal, 2006: 2), conceptual, sistemática (Rodríguez y Acosta, 2007: 41), hermenéutica (Ramírez, 2006: 11), de construcción interdisciplinaria (Escuela Latinoamericana de Cooperación y Desarrollo, 2005: 30) y de producción continua (Aon y Vampa, s. f.; Rodríguez y Acosta, 2007: 44-45).

\section{EN BUSCA DE ALGUNAS DEFINICIONES DEL ESTADO DEL ARTE COMO UNA MODALIDAD DE INVESTIGACIÓN CIENTÍFICA}

El proceso de teorización del estado del arte como una modalidad de investigación sigue abierto. Aunque es creciente el número de campos del saber y temas en los que se practica, debido a la novedad de esta metodología en la investigación filosófica parece pertinente una especulación que sirva como referente para la interpretación y valoración de lo que, desde algunos esquemas epistemológicos, podría parecer atípico, incluso antitético, o confundirse con la etapa compilatoria propia de la aplicación de otra metodología de investigación científica.

4 La autora afirma: "El análisis de los datos es un proceso en continuo progreso, la recolección y el análisis de los datos van de la mano, esto va completando el espiral en el trabajo de campo" (Diez, 2003: 329-330). 
Parece deducible que el objeto material del estado del arte es la producción intelectual y que su objeto formal es la expresión de contenido, especulativa y de aplicabilidad, como totalidad, de dicha producción. Esto puede ser coherente con que se afirme "[...] en el Estado del Arte se habla del conocimiento de punta o ultima $[$ sic $]$ generación” (Universidad de la Sabana, s. f.).

\section{Propuesta de dos nuevas definiciones del estado del arte como UNA MODALIDAD DE INVESTIGACIÓN CIENTÍFICA}

Para desarrollar este subtema se estudiaron 64 textos, de los que se descartaron 17. Se proponen las siguientes definiciones de estado del arte como referente para el análisis de las nociones halladas y como parte de los productos de la especulación durante el trabajo realizado en esta investigación:

Abordaje, proceso y resultado sistemáticos de la generación y actualización de la compilación de productos intelectuales, analizando su perfil especulativo y la integración de éstos, sus actores y gestores.

Modalidad investigativa con la que se produce, como efecto de un proceso compilatorio y de un estudio especulativo, un perfil crítico documental actual.

Los términos clave para la categorización de estas definiciones se concretan, respecto a la primera propuesta, como abordaje sistemático - proceso sistemático - resultado sistemático - generación de la compilación de productos intelectuales - actualización de la compilación de productos intelectuales - análisis del perfil especulativo de productos intelectuales - integración de productos intelectuales - análisis de actores de productos intelectuales - análisis de gestores de productos intelectuales.

Respecto a la segunda tenemos producir - proceso compilatorio - estudio especulativo - perfil crítico documental actual.

La distribución de términos de ambas definiciones propuestas, según su orden de inserción en este proceso investigativo, es la siguiente: proceso sistemático - abordaje sistemático - estudio especulativo - proceso compilatorio - generación de la compilación de productos intelectuales - actualización de la compilación de productos intelectuales - integración de productos intelectuales - análisis de actores de productos intelectuales - análisis de gestores de productos intelectuales - análisis del perfil especulativo de productos intelectuales - resultado sistemático - producir - perfil crítico documental actual. 
En el siguiente análisis se exponen entre corchetes los fragmentos de las definiciones arriba propuestas, que parecen tener relación con las halladas en otras fuentes, y algunos comentarios.

Según Vélez y Galeano (2002: 1), un estado del arte "es una investigación sobre la producción -investigativa, teórica o metodológica- existente acerca de determinado tema [Productos intelectuales] para develar [Identificación], desde ella, la dinámica y lógica presentes en la descripción, explicación o interpretación que del fenómeno en cuestión hacen los teóricos o investigadores". [Caracterización]. [Falta la integración de éstos, sus actores y gestores].

Martínez (1999: 13) dice:

El Estado del Arte pretende recuperar reflexivamente las distintas lecturas (escrituras) [Respecto a "escrituras": se relaciona con la actualización de la compilación de productos intelectuales, aunque parece quedar faltando los registrados en los demás medios] que se han venido produciendo sobre un determinado aspecto de la realidad social [No sólo sobre esta, sino también acerca todo lo real o con todo lo real], para avanzar en la comprensión del complejo entramado de conocimientos [Identificación] y que desde posturas epistemológicas, teóricas y metodológicas [Caracterización] intentan dar cuenta del hecho social particular en estudio. [Integración de lo leído, sus autores y de quienes interactúan en y con estos productos intelectuales].

El estado del arte es, también:

[...] el recorrido que se realiza -a través de una investigación de carácter bibliográfico- [Abordaje, proceso y resultado] con el objeto de conocer y sistematizar la producción [Esa sistematización puede hacerse sobre la actualización de la compilación de productos intelectuales y la identificación, caracterización e integración de éstos, sus actores y gestores] científica en determinada área del conocimiento. [Se refiere a actualización; falta generación; menciona sólo la producción científica, reduciendo así el objeto material de los Estados del arte.] Esta exploración documental trata de elaborar una lectura de los resultados alcanzados en los procesos sistemáticos de los conocimientos previos a ella. (Souza, 2008: 1) 
En otra fuente se lee:

En definitiva, es una lectura crítica [Integración de los productos intelectuales, sus actores y gestores] y analítica [Identificación, caracterización] de los trabajos significativos [De esta expresión no se deduce necesariamente que esté haciendo referencia al abordaje, proceso y resultado sistemáticos de la generación y actualización; la selección de los trabajos significativos es posterior, más bien producto de contar con un buen Estado del Arte.] ya realizados sobre ese tema [Hace referencia a la actualización, pero no a la generación.], que permite conocer cómo fue tratado y las posturas hegemónicas en ese campo. (Aon y Vampa, s. f.)

Mientras que en una de 2007:

[...] un Estado del Arte es una modalidad de investigación sobre el conocimiento producido [Productos intelectuales], acumulado y circulante en distintos formatos bibliográficos, los que se localizan [Abordaje], registran y delimitan en una base documental [Compilación], sobre la cual se realiza un proceso [Proceso] de clasificación [Sistemáticos] en un nivel descriptivo [Identificación] y categorización en un nivel conceptual [Caracterización], que en su confluencia permite analizar las dimensiones, relaciones y posiciones estratégicas que caracterizan y constituyen un campo de saber. [Esta última idea podría relacionarse con las de generación y actualización del Estado del Arte.] (Rodríguez y Acosta, 2007:39)

En una institución de carácter internacional: “[...] clasificar y sistematizar [Abordaje, proceso y resultado sistemáticos] enfoques” (Cinterfor/OIT, s. f.: 6).

En un estado del arte que abarca 15 años:

Como estrategia para la investigación, un Estado del Arte es la recopilación exhaustiva y sistemática de las fuentes disponibles [Abordaje, proceso y resultado sistemáticos] acerca de un tema determinado [De productos intelectuales]. Es como "poner orden en la casa" para conocer qué tanto se ha hecho [Identificación], cómo se ha procedido, cuáles son sus avances, las principales teorías, problemas o consideraciones que han tenido lugar desde que se empezó a tratar. [Caracterización e integración de éstos, sus actores y gestores]. Consiste en una técnica de búsqueda y orientación al servicio de la gestión del conocimiento que permite, a cualquier persona interesada en un tema, acercarse a sus diferentes enfoques, métodos y logros. (Escuela Latinoamericana de Cooperación y Desarrollo, 2005: 8)

En una universidad: "El Estado del Arte, que es todo un género, es una instancia de puesta al día y recopilación de lo que se ha estudiado acerca de un determinado tema" (Universidad Torcuato di Tella, s. f.: 1) si bien no 
especifica qué entiende por género. Finalmente, en una investigación chilena: "Presentación de los antecedentes de investigaciones previas disponibles en la literatura especializada en el tema a desarrollar o el "Estado del Arte” respecto al problema” (Universidad Andrés Bello, 2005: 13). Cabe acotar que, aunque la abarca, el estado del arte es una noción más amplia que la de investigaciones previas disponibles.

\section{Comentarios A LO QUE ALgUnOS DiCEN QUE NO ES UN ESTADO DEL ARTE}

Lo nuclear:

[...] su esencia es la de "ir tras las huellas" del tema que interesa investigar. [Ir tras las huellas es lo que se hace en la etapa compilatoria de la mayoría de las investigaciones. El Estado del Arte brinda, de modo más abarcante pero, por limitaciones humanas, no absolutamente completo, la actualidad de los productos intelectuales que podrían dejar huella]. (Universidad de Antioquia, s. f.: 1)

\section{La precedencia:}

Es una de las primeras etapas que debe desarrollarse dentro de una investigación. [Esto parece corresponder más a la etapa compilatoria de una investigación en la que se debería contar siempre con el Estado del Arte más completo posible y, si no lo hay y aún es tiempo de elegir el tema de investigación, sería preferible comenzar por hacerlo. Tampoco es una compilación sin investigación crítica, de lo contrario no se diferenciaría de una base de datos bien hecha]. (Universidad de Antioquia, s. f.: 1)

\section{La representatividad:}

[...] el autor hace un análisis [...] para ello describe ampliamente el problema, basado en saberes [...] esto lo conduce al planteamiento de la hipótesis, para cuya demostración se anda en el análisis de un corpus, suficientemente representativo y en los aportes teórico-prácticos que otros autores han hecho sobre el tema en cuestión. Este análisis es, precisamente, el Estado del Arte. [Parece que se está confundiendo lo que es el Estado del Arte con lo que podría ser parte de una estructura que puede ser aplicada en diferentes modalidades de investigación: planteamiento del problema - etapa compilatoria de los aportes teóricos y prácticos de otros autores sobre el tema en cuestión, no lo más completo posible, sino que basta que sea "suficientemente representativo" - planteamiento de la hipótesis análisis - demostración de la hipótesis]. (Universidad de Antioquia, s. f.: 3 ) 
La construcción del objeto de estudio (Pontificia Universidad Javeriana, s. f.: 1). [El Estado del Arte es tanto el abordaje como el proceso y el resultado de la ejecución de una modalidad investigativa que recibe ese mismo nombre, y su producto se constituye en una herramienta fundamental para la identificación más precisa de las características propias y del entorno del objeto de estudio en la mayoría las investigaciones].

Esta exploración documental trata de elaborar una lectura de los resultados alcanzados en los procesos sistemáticos de los conocimientos previos a ella. (Souza, s. f.: 1) [También la investigación así descrita requiere una compilación de información sobre la modalidad investigativa "Estado del Arte"]. "[...] es cuantitativamente diferente a una simple recopilación y ordenamiento del tema en cuestión. [Es así, por el estudio crítico que se hace a la documentación compilada]" (Ramírez, 2006: 12).

\section{Sentido del estado del arte}

Se expone algunos referentes acerca del sentido del estado del arte como modalidad de investigación científica y luego se hace un comentario general sobre ellos.

"El objetivo de producir un Estado del Arte, es conocer los diferentes enfoques que caracterizan a distintos modelos". (República Argentina y Diamant, s. f.)

"[...] cuya finalidad es dar cuenta del sentido del material documental sometido a análisis" (García, s. f.: 2).

"[...] aporta conocimiento acerca de una temática elegida para empezar a formular el problema de investigación”. (Aon y Vampa, s. f.)

"[...] nace la escasez de estudios que den cuenta de lo que se viene haciendo en el campo [...] paso previo al diseño de estrategias para orientar las acciones futuras" (Unesco, 2005: 5, 7)

"[...] obtener una base de datos e informaciones más concretas que permitieran recomendar [...] acciones específicas para el mejoramiento" (Unesco, 2005:7)

"[...] Aportar una solució a la] "Ausencia de suficiente fondo de investigación”. (Fernández, 2006: 8) 
“Revisión crítica de la literatura: ¿cuál es el Estado del Arte?”. (Nupia, 2008: 1)

La necesidad:

[...] Necesitamos saber mas [sic] acerca de lo que está pasando en las diferentes "periferias" (nacionales, disciplinares, de medios) para aprender sobre las raíces conceptuales de nuestro presente y actuar en una academia globalizada futura, abriendo nuestras mentes a la fascinante diversidad (y cunidad?) de nuestras memorias, imágenes, estilos, focos, estrategias y mundos - de - vida como investigadores cualitativos. (Katja, Cisneros y Faux, 2005: 1)

"En particular, se requiere fortalecer la capacidad investigativa de las instituciones en los problemas y procesos que se relacionan con su propia naturaleza”. (Martínez y Vargas, 2002: 8)

“[...] "Estado del Arte", es decir, una etapa en el proceso de avance en la solución de cierto tipo de problemas, y por lo tanto algo susceptible de mejora. Pero para mejorar hay que ir constantemente a los principios, revisarlos y replantear las soluciones”. (Enrique, 2008)

El estado del arte ayuda a identificar, entre otras cosas, "¿Qué se produce? ¿Dónde? ¿Quiénes y cómo? ¿Para qué?”. (Franco et al., 2007: 4)

Se concluye que lo que se describe como efecto de esta modalidad investigativa coincide con la razón de ser del trabajo científico: abrir la mente, contextualizar, identificar, revisar críticamente, fortalecer la capacidad investigativa, aprender, conocer, diseñar estrategias, diversificar, actuar, obtener, recomendar, mejorar, avanzar, dar cuenta, saber, aportar fondo de investigación, globalizar y solucionar, entre otros motivos.

\section{Aporte CiEnTífico DEL ESTADO DEL ARTE}

Ofrece elementos que enriquecen el conocimiento; aporta elementos para "identificar y cuestionar las insuficiencias y excesos de autores [También para reconocer lo que parece ser sus aportes y los de los gestores de la información y del conocimiento]". (Cruz et al., s. f.: 2)

La experiencia del rastreo de la producción del conocimiento "[...] permitió afinar un modelo que intenta trascender la catalogación y síntesis tipo abstract de las investigaciones, para apostar a un análisis más profundo, que de [sic] cuenta del tratamiento temático y de las nociones [...] que subyacen, se configuran y enuncian en este tipo de conocimiento". (República de Colombia, GTZ y Unicef, 2004: 7) 
"Desde la construcción del Estado del Arte se pretende recuperar la información reflexiva de las diversas lecturas que se han generado del tema [...], para avanzar en la comprensión del entramado de conocimientos" (Ramírez, 2006: 12).

"Este Estado del Arte da cuenta de las investigaciones [...] que tuvieron lugar "principalmente" durante [...], aunque hemos incluido algunas investigaciones desarrolladas durante [en el Estado del Arte consta la fecha hasta la que actualiza]" (Messina, 1999: 2). ${ }^{5}$

"Aunque no se trata de una tarea neutral y aséptica, demanda ajuste a los procedimientos propios de esa revisión. Y, por supuesto, demanda asumir una mirada de conjunto que atribuya un sentido al trabajo previo, del que se parte y sobre el que se asume la propia voz en una materia" (Universidad Torcuato di Tella, s. f.).

Se concluye que, con base en el estado del arte, se puede identificar el perfil de quien o quienes producen y gestionan los productos intelectuales y las características generales de estos productos, de modo que se aportaría una mirada prospectiva sobre autores, gestores y obras. Asimismo, sirve de base para

[...] compilar productos intelectuales, conocer a sus autores y gestores, investigarlos, fundar y dar continuidad a líneas de investigación que aporten conocimiento: «Este segundo Estado del Arte, se entiende como un proyecto que da continuidad a líneas de investigación, tanto metodológicas como analíticas, lo que no significa un intento de "replicar" lo encontrado en el primer esfuerzo» (República de Colombia, GTZ y Unicef, 2004: 7, 43)

Es recurso a modo de ruta de navegación:

Un Estado del Arte es un mapa que nos permite continuar caminando; un Estado del Arte es también una posibilidad de hilvanar discursos que en una primera mirada se presentan como discontinuos o contradictorios. En un Estado del Arte está presente la posibilidad de contribuir a la teoría y a la práctica de algo, en este caso de la formación docente (Messina, 1999: 1).

Es un medio para el conocimiento, la socialización y aplicación acertada de la actualidad: "Estos congresos, además de presentar el Estado del Arte de la EIB [...]" (Abram, 2004: 4). "En un Estado del Arte está presente la posibilidad de contribuir a la teoría y a la práctica de algo". (Messina, 1999: 2)

5 El trabajo de Messina fue publicado como la tercera parte del libro de Lagos, Rivera y Kraushaar (2002). 
Concreción de criterios: "[...] exponemos los criterios de localización, selección y registro de la información, a partir de los cuales se diseñó la base de datos que sirvió de soporte documental para el análisis" (Rodríguez y Acosta, 2007:2).

Rastreo: “[...] rastrear la producción de conocimiento". (República de Colombia, GTZ y Unicef, 2004: 7, 43)

Recopilación: "[...] recopilar los saberes". (Cruz et al., s. f.: 2) "[...] recoge una colección de [...] presenta una serie de estudios [...] se complementa con un estudio sobre [...]" (Unesco, 2005: 5). "La información y la documentación obtenidas fueron recopiladas en el presente volumen" (Unesco, 2005: 7).

Revisión: "Revisar de manera detallada y cuidadosa los documentos que tratan dicho tema" (García, s. f.: 2).

Clasificación: "[...] clasifica [...] las diferentes fuentes de información" (Cruz et al., s. f.: 2).

Ordenación: "[...] ordenar y organizar la información recabada de un conjunto amplio de textos en su complejidad y detalle" (Cruz et al., s. f.: 2). "Un Estado del Arte [...] organiza los resultados de la investigación reciente. [No sólo los resultados de la investigación reciente: si así fuera, no se podría organizar la producción intelectual como aporte para la primera investigación]" (Universidad de Antioquia, s. f.: 1). Profundización en el análisis de las fuentes: "[...] profundiza en el análisis de las fuentes de información" (Cruz et al., s. f.: 1). "[...] analiza lo que se dice de lo que se ha dicho" (Cruz et al., s. f.: 1). "[...] revisa si los textos proyectan [...] tal como ella es o a una falsa [...] que se refuerza por el imaginario social. Qué se dice, quién lo dice y lo que no se ha dicho" (Cruz et al., s. f.: 2). "[...] el autor hace un análisis" (Universidad de Antioquia, s. f.: 3).

Recurso de crítica y análisis:

En definitiva, es una lectura crítica y analítica de los trabajos significativos ya realizados sobre ese tema [Esta lectura se hace dentro de la investigación denominada Estado del Arte, como en las demás, pero en ella no se agota lo que es esta modalidad, que incluye también la etapa compilatoria], que permite conocer cómo fue tratado y las posturas hegemónicas en ese campo. (Aon y Vampa, s. f.)

"[...] describe el marco analítico - político general [...] se complementa con un estudio sobre [...] y una amplia bibliografía comentada que da fe de las tendencias actuales de la investigación en el tema [es una muestra entre los variados modos como se puede trabajar un estado del arte]". (Unesco, 2005: 6) 


\section{Algunos procesos de indagación implicados EN LA APLICACIÓN DE LA METODOLOGÍA DEL ESTADO DEL ARTE}

Utilizar: "[...] utiliza los textos como primera expresión del objeto" (Cruz et al., s. f.: 2). "[...] los trabajos significativos ya realizados sobre ese tema" (Aon y Vampa, s.f.).

Resumir: un estado del arte "[...] resume [...] los resultados de la investigación reciente" (Universidad de Antioquia, s. f.: 1). "[...] resumir" (Manrique y Zapata, 2006: 13). "[...] resume [...] los resultados de investigación reciente en una forma novedosa que integra y agrega claridad al trabajo en un campo específico. El artículo asume un conocimiento general del área" (González, s. f.: 18).

Evaluar: "El artículo enfatiza la clasificación de la literatura existente, desarrollando una perspectiva del área y evaluando las principales tendencias" (González, s. f.: 18). "[...] evaluar" (Manrique y Zapata, 2006: 13).

Cuestionar: "[...] cuestionar" (Manrique y Zapata, 2006: 13).

Criticar: "[...] consiste en [...] criticar" (Manrique y Zapata, 2006: 13).

Clarificar: "[...] integrando y agregando de forma novedosa claridad al trabajo en un campo específico" (Universidad de Antioquia, s. f.: 1).

Develar: "[...] develar" (Universidad de Antioquia, s. f.: 1).

\section{Algunos productos Del estado del arte en una tesis}

Una tesis se plantea, como uno de sus objetivos centrales, contribuir a la construcción de nuevos conocimientos en alguna disciplina o campo del saber; esto supone, de alguna manera, el conocimiento de lo ya existente en la materia que se va a trabajar, dado que no se puede procurar aportar nuevos conocimientos si no se tiene una idea acabada respecto de los que ya se produjeron. En este sentido, dar cuenta del Estado del Arte significa explicar qué se ha investigado hasta ahora en relación con nuestro tema específico de estudio, intentando distinguir, además, el modo en que nuestra investigación puede significar un enriquecimiento de los conocimientos existentes y no una mera reiteración de estudios anteriores. Esto último redundará sin duda en una mejor precisión del problema y en la formulación de preguntas de investigación apropiadas. Por otro lado, esta revisión documental nos permite conocer si existen marcos teóricos, datos empíricos o formulaciones conceptuales sobre el tema que estamos estudiando. También nos proporciona información sobre metodología, diseños instrumentales más utilizados y técnicas de recogidas de datos, lo que facilitará la identificación y comprensión de procedimientos de investigación. (Souza, s. f.: 1) 


\section{Otros IMPACTOS DEL ESTAdo DEL ARTE}

"Su análisis ha contribuido sustancialmente a elaborar un marco básico para comprender las tendencias principales de la realidad y la práctica de [...] en la región en relación con [...]”. (Unesco, 2005: 7)

"La integración de la investigación ha sido calificada como uno de los componentes principales de [...], como un programa permanente que intente contribuir con el conocimiento y que sea una herramienta para ayudar a diseñar estrategias que valoren la diversidad y que promuevan una distribución equitativa de oportunidades [...]”. (Unesco, 2005: 25)

"Áreas prioritarias para la investigación han sido definidas". (Unesco, 2005: 25)

"La falta de información para la toma de decisiones parece estar en el centro de los problemas definidos". (Unesco, 2005: 25)

"[...] las áreas en donde se necesita expansión [...] 1) la formación de [...], 2) la investigación sistemática de [... 3) el fortalecimiento de las redes de información, y 4) la construcción y el desarrollo de indicadores que ayuden a comprender los logros así como las dificultades de [...] en [...]”. (Unesco, 2005: 25)

Correspondencia con la necesidad de un trabajo sistemático:

El primer esfuerzo exploratorio para el desarrollo de un "Estado del Arte" en [...] muestra una diversidad de experiencias que no son fácilmente comparables. Esto se debe principalmente a las limitaciones que ya se han subrayado en el proceso de [...], especialmente en lo que concierne a la falta de investigación sistemática y documentación [...] la información de naturaleza estadística no está preparada, disponible o compilada en forma pertinente. [...] construir un marco descriptivo analítico que [...] ayude a sugerir orientaciones básicas dentro de las líneas de acción y en el marco de los [...] temas prioritarios propuestos para [...]. (Unesco, 2005:33)

\section{Conclusiones}

Se ha ilustrado que la modalidad investigativa denominada estado del arte facilita aciertos, evita errores y clarifica el abordaje de la investigación de cualquier tema, época, autor, obra o escuela, también respecto al campo filosófico, además de garantizar que, con el aporte de la construcción de elementos que complementen el perfil crítico documental actual, se abarca 
al menos la mayor parte del material objeto de estudio y de la producción intelectual acerca de un autor, su obra, los estudiosos y gestores de la misma, siendo así una modalidad investigativa que sirve de soporte para las demás en cualquier campo del saber.

Con lo hasta ahora expuesto se ha intentado identificar y reconocer el aporte de la modalidad de investigación científica denominada estado del arte en el contexto de la innovación científica, filosófica y de la historia del desarrollo del conocimiento que pueda redundar en beneficio del ser humano y de la naturaleza. Parece razonable concluir que el estado del arte es una exigencia universal, desde las perspectivas transtemporal y transcultural, que depende en buena parte del aprovechamiento de los medios de comunicación. Cualquier mejora honesta en el modo de recoger lo que se sabe acerca de lo que se quiere indagar supone un excelente recurso para avanzar en el conocimiento de lo real y para difundirlo.

El deseo de trabajar con rigor en las fuentes cuando no estaba tan difundida esta metodología se observa en la obra René Descartes: Discurso del método de Carlos Cardona Pescador, ${ }^{6}$ en la que refleja ser consciente de que el trabajo del investigador demanda un continuo replanteamiento de las metodologías y resultados del rastreo documental y testimonial que facilita la calidad de los contenidos que utiliza para su contemplación, de los que depende, en parte, que sepa responder a la alta exigencia de producción y socialización del conocimiento en el contexto de las estrecheces de tiempo a que están sometidas frecuentemente actividades como la académica e investigativa, con sus exigencias propias de calidad en los productos de conocimiento, proporcionadas al aprovechamiento de los medios a que se tenga acceso, y de su pronta universalización, que ojalá también se aplique a las mismas metodologías y resultados de compilación, acelerando de este modo los procesos culturales, en este caso, en la filosofía y desde este campo del saber.

Del tratamiento sistemático de las conceptualizaciones de estado del arte que se han tenido en cuenta en esta investigación se ha concluido la conveniencia de hacer una reflexión más profunda acerca de las definiciones de estado del arte y sus aplicaciones, de las que también depende la calidad de los aportes de la comunidad científica al pleno desarrollo humano personal,

6 Por ejemplo: "Se sigue aquí el texto publicado en el tomo VI de las Oeuvres complètes, edición Adam-Tannery, reproducido fotostáticamente en la publicación dirigida y anotada por E. Gilson: René Descartes, Discours de la méthode, texte et commentaire, 4 éd., Librairie philosophique Vrin, Paris, 1967. La indicación de página y líneas de cada texto citado del Discurso, corresponden a esa edición. Para la versión castellana hemos utilizado fundamentalmente la traducción de García Morente: Discurso del método, colección Granada, Ed. Granada, Madrid, s. f., con algunos retoques a la vista del original francés y en consideración del castellano de hoy". (Cardona, 1987: 7) 
familiar y social, integral y sostenible, en todos los saberes conformados con indagaciones y corroboraciones que, por sistemáticas, deben partir de la mayor aproximación posible a la totalidad de lo conocido sobre un aspecto de la realidad.

\section{REFERENCIAS}

Abram, M. L. 2004. Estado del Arte de la educación bilingüe intercultural en América Latina. Fecha de consulta: 12 de diciembre de 2007. http:/www.iadb.org/sds/ doc/ind-mabrams.pdf

Acosta, M. R.. 2005. "Tragedia y filosofía de la historia: Schiller, Hölderlin y la recuperación de lo clásico”. Proyecto de tesis doctoral, Universidad Nacional de Colombia. Fecha de consulta: 31 de mayo de 2009. http://www.filosofiaytragedia. com/textos/bibliografia.html

Alba, E. y C. Cotta. S. f. Docencia sobre Algoritmos Evolutivos en la Universidad de Málaga. España: Universidad de Málaga, Departamento de Lenguajes y Ciencias de la Computación. E.T.S. Ingeniería Informática. Fecha de consulta: 20 de febrero de 2015. http://www.lcc.uma.es/ eat/pdf/maeb04e.pdf

Aon, L. y M. S. Vampa. S. f. El Estado del Arte y la memoria. Fecha de consulta: 20 de febrero de 2015. http:/perio.unlp.edu.ar/seminario/nivel2/nivel3/ponencias/ mesa9/AON_VAMPA.htm

Benavides, F. y F. Pedró. 2007. "Políticas educativas sobre nuevas tecnologías en los países iberoamericanos”. Revista Iberoamericana de Educación 45 (septiembre-diciembre): 19-69. Fecha de consulta: 20 de febrero de 2015. http://www.rieoei. org/rie45a01.pdf

Cardona, C. 1987. René Descartes: Discurso del método, Colección Crítica Filosófica, 1. $3^{\mathrm{a}}$ ed. Madrid: Magisterio Español.

Cinterfor/OIT (Centro Interamericano para el Conocimiento en la Formación Profesional). S. f. Estrategias de educación y formación para los niños y jóvenes desfavorecidos en América Latina. Fecha de consulta: 12 de diciembre de 2007. http:// www.ilo.ch/public/spanish/region/ampro/cinterfor/temas/youth/exp/unesco/ iii/index.htm

CINDOC-CSIC. 2004 (octubre). Revistas cientificas electrónicas: Estado del Arte. Fecha de consulta: 20 de febrero de 2015. http://pendientedemigracion.ucm.es/info/eurohum/docs/e-revistas_informe.pdf

Corvalán, Graziella. 1985. Estado del Arte del bilingüismo en América Latina. Chile: Centro de Investigación y Desarrollo de la Educación (CIDE). Fecha de consulta: 5 de junio de 2009. http://biblioteca.uahurtado.cl/cgi-bin/wxls.exe

Cruz Toral, Dinora Edith, Paz Diéguez Delgadillo, Sonia Macias Lavin y Exekiel Obregón Álvarez. S. f. Explicar la estrategia utilizada por el autor para construir el Estado del Arte del problema que aborda. Fecha de consulta: 14 de mayo de 2009. http://desinuam.org/salon/2007p/UAMX-teoria/Eq2-Ac3.doc

Diaz, A. y A. Reyes. 2004. Realidad virtual y presencia. Curso. Universidad de Málaga. Fecha de consulta: 20 de febrero de 2015. http://griho2.udl.es/i2004/tut/antarc.pdf 
Díez, M. A. 2003. “Teorías y creencias pedagógicas de los profesionales sin formación docente que enseñan en los Institutos Superiores de Profesorado de la Proviencia de Santa Fé. Estado del Arte del paradigma del pensamiento del profesor", en Docentes que hacen investigación Tomo 2, Jorge Cardelli y Miguel Duhalde, comps., 329-344. Buenos Aires, Argentina: Miño y Dávila Editores.

Enrique. 2008 (julio 5). "No habría Shrek sin matemática”, GuateCiencia blog. Fecha de consulta: 20 de febrero de 2015. https:/guateciencia.wordpress. com/2008/07/05/no-habria-shrek-sin-matematica/

Escuela Latinoamericana de Cooperación y Desarrollo. 2005. Cooperación para el desarrollo Unión Europea-América Latina. Fuentes documentales en las áreas de política y metodologías 1990-2005. Fecha de consulta: 20 de febrero de 2015. https://www.academia.edu/5901095/Cooperación_para_el_desarrollo_Unión_ Europea-América_Latina_fuentes_documentales_en_las_áreas_de_pol\%C3\% ADtica_y_metodolog\%C3\%ADas_1990-2005

Fernández, P. 2006. Valoración urbanística de empresas aplicado [sic] al estudio de los daños y perjuicios por traslado y cese de actividad económica en actuaciones urbanísticas y expropiatorias. Proyecto de tesis. Programa de gestión y valoración urbana de la Universitat Politecnica de Catalunya, España. Fecha de consulta: 20 de febrero de 2015. http://www.cpsv.upc.es/tesis/PT06_PabloFernandez.pdf

Franco, M. C. 2007. La Producción Académica en Educación Social y Comunitaria en Bogotá, D.C.: Un Estado del arte. Fecha de consulta: 2 de noviembre de 2007. http://sabanet.unisabana.edu.co/educacion/maestrias/maestria/presentaciones/ Estado\%20del\%20arte.pdf

García, B. E. S. f. Estado del Arte o estado del conocimiento. Fecha de consulta: 20 de febrero de 2015. http://www.google.com/url? sa=t\&rct=j\&q=\&esrc=s\&source=web \&cd=1\&ved=0CB8QFjAA\&url=http $\% 3 \mathrm{~A} \% 2 \mathrm{~F} \% 2 \mathrm{Fcmap} . u p b . e d u . c o \%$ 2Frid\%3D1191453502046_1854553289_1135\%2FESTADO\%2520DEL\%2520ARTE.ppt\&ei=jqznVJ_vD9LlsAStpoD4CQ\&usg=AFQjCNGB07WZSXDol2633OwdTT1RI2RZcQ\&sig2=A0nhvxSsOAoXHvA4grd6gQ\&bvm=bv.86475890,d.cWc

Gobierno de España. Ministerio de Educación Cultura y Deporte. S. f. Tesis doctorales TESEO. Fecha de consulta: 20 de febrero de 2015. https://www.educacion. gob.es/teseo/mostrarSeleccion.do

González, F. A. S. f. Definición de Tema de Investigación, Estado del Arte y Evaluación de Artículos. Colombia: Universidad Nacional de Colombia, Depto. Ing. de Sistemas e Industrial, Seminario de Investigación. Fecha de consulta: 20 de febrero de 2015. http://dis.unal.edu.co/ fgonza/courses/2004-I/seminario/survey_review.pdf

Hernández, R., C. Fernández y P. Baptista. 2010. Metodología de la investigación, 5 a. ed., México: McGraw Hill.

Katja, C. A., C. A. Cisneros y R. Faux. 2005. "Sobre los centros y periferias de la investigación cualitativa”. Forum Qualitative Social Research 6 (3) (septiembre): art. 49. Fecha de consulta: 20 de febrero de 2015. http://www.qualitative-research. net/index.php/fqs/article/view/2/5

Lagos, M. H., C. Rivera y L. Kraushaar, eds. 2002. Formación profesional docente: tendencias \& experiencias. Santiago de Chile: Universidad de Los Lagos, Red de Servicios Universitarios Regionales. Fecha de consulta: 15 de mayo de 2009. http://www.observatoriopoliticaspublicas.cl/s_semestrales/seminario3\% 5B1\%5D.pdf 
Leal, E. 2006. Situación actual de las innovaciones pedagógicas, organizacionales y tecnológicas en la Educación Básica venezolana. Período 1986-2004. Venezuela: UNA. Fecha de consulta: 31 de mayo de 2009. http://eduardoleal.net/content/blogsection/0/9/

López Cerezo, J. A. 2002. "Ciência, Tecnologia e Sociedade: O estado da arte na Europa e nos Estados Unidos”, en L. Woellner, E. Yoshie, P. Varela y D. F. Cargano, eds. Ciência, Tecnologia e Sociedade: O desafio da interação. IAPAR: Londrina: 3-39.

Manrique, M. P. y C. V. Zapata. 2006. Estado de arte desde una perspectiva social sobre las condiciones de las viviendas donde se acentúa la población desplazada en Bogotá en el periodo del 2000 al 2006. Colombia: Universidad Colegio Mayor de Cundinamarca, Facultad de Ciencias Sociales. Fecha de consulta: 12 de diciembre de 2007. http://www.minproteccionsocial.gov.co/entornoambiental/library/documents/DocNewsNo15400DocumentNo2618.PDF

Martínez, L. A. 1999 “¿Qué significa construir un Estado del Arte desde una perspectiva hermenéutica?” Criterios (Pasto) 8: 13-20.

Martínez, E. y M. Vargas. 2002. La investigación sobre la educación superior en Colombia. Un estado del arte. Bogotá: Instituto Colombiano para el Fomento de la Educación Superior ICFES. Fecha de consulta: 31 de mayo de 2009. http:// joomla.icfes.gov.co/web/index.php?option=com_docman\&task=doc_download \&Itemid $=307 \&$ gid $=1454$

Messina, G. 1999. "Investigación en o investigación acerca de la formación docente: un Estado del Arte en los noventa”. Revista Iberoamericana de Educación 19 (enero-abril).Fecha de consulta: 20 de febrero de 2015. http://www.rieoei.org/oeivirt/ rie19a04.htm

Monroy, S. E. 2004. "Del fraude, el método inductivo y los artículos científicos. Una réplica a Peter Medawar". Revista Colombiana de Filosofía de la Ciencia (Universidad El Bosque, Bogotá) 3 (10- 11): 41- 48. Fecha de consulta: 20 de febrero de 2015. http://www.redalyc.org/articulo.oa?id=41401102

Nupia, O. 2008. Seminario de tesis. PEG. Universidad de los Andes. Facultad de Economía, Bogotá. Fecha de consulta: 20 de febrero de 2015. https://economia. uniandes.edu.co/images/archivos/pdfs/Programas/Maestria_economia_ peg/2008-10/SeminariodeTesisPeg_OscarNupia_200810.pdf

Pontificia Universidad Javeriana. S.f. El estado del arte. La construcción del objeto de estudio. Fecha de consulta: 12 de diciembre de 2007. http://www.javeriana.edu.co/ Facultades/Teologia/servicios_formacion_int_univ/ecoteo1/pyf-estar.pdf

Posada, N. L. 2011. Carlos Cardona: Estado del Arte. España: Universidad de Málaga.

Ramírez, A. Y. 2006. Aproximación al concepto del retraso mental leve en Colombia. Medellín: Universidad Pontificia Bolivariana, Escuela de Educación y Pedagogía, Facultad de Educación. Fecha de consulta: 20 de febrero de 2015. http://www. google. $\mathrm{com} /$ url? $\mathrm{sa}=\mathrm{t} \& \mathrm{rct}=\mathrm{j} \& \mathrm{q}=$ \&esrc $=\mathrm{s} \&$ source $=$ web $\& \mathrm{~cd}=1 \& \mathrm{ved}=0 \mathrm{CCQQF}$ jAA\&url=http\%3A\%2F\%2Fwww.loracep.org\%2Fweb\%2Findex.php\%3Foption\%3Dcom_docman\%26task\%3Ddoc_download\%26gid\%3D619\%26Itemid\%3D\&ei=iKvnVMCPG4rbsASuuYGQAQ\&usg=AFQjCNFvPc67Arg7xik826gU_ cHvk_5fLQ\&sig2=f3IuI4yOU1G02VceAFx73w\&bvm=bv.86475890,d.cWc

República Argentina, Ministerio de Cultura y Educación y A. E. Diamant. S. f. Propuesta para un análisis comparativo de instrumentos de evaluación de logros académicos. Fecha de consulta: 14 de mayo de 2009. http://www.ince.mec.es/cumbre/ d1-04.htm 
República de Colombia, GTZ y Unicef. 2004. Estado del Arte del conocimiento producido sobre jóvenes en Colombia 1985-2003. Fecha de consulta: 20 de febrero de 2015. https://semillerojovenes.files.wordpress.com/2010/07/informe-estado-del-arte-sobre-jovenes-1985-2003.pdf

Rodríguez, S. P. y W. A. Acosta. 2007. "La emergencia de la didáctica de las ciencias sociales: ¿campo en consolidación o en disgregación?” Folios 25 (segunda época, primer semestre): 37-52. Fecha de consulta: 15 de mayo de 2009. http://www. scielo.org.co/pdf/folios/n25/n25a03.pdf

La construcción del objeto de estudio. S. f. Fecha de consulta: 20 de febrero de 2015. http://www.javeriana.edu.co/Facultades/Teologia/servicios_formacion_int_ univ/ecoteo1/pyf-estar.pdf

Soto, G. 2007 Filosofía medieval. Bogotá: Universidad Pedagógica Nacional-San Pablo.

Souza, M. S. S. f. El Estado del Arte. Fecha de consulta: 12 de diciembre de 2007. http://perio.unlp.edu.ar/seminario/nivel2/nivel3/el\%20estado\%20del\%20arte_ silvi na_souza.pdf

Souza M. S. 2008. La centralidad del estado del arte en la construcción del objeto de estudio 2. Fecha de consulta: 20 de febrero de 2015. http://perio.unlp.edu.ar/seminario/nivel2/nivel3/textos_actualizados_2008/La\%20centralidad\%20del\%20 estado\%20del\%20arte\%20en \%20la\%20construccin \%20del $\% 20$ objeto $\% 20$ de\%20estudio.pdf

Torregoza, E. J. 2006. "Pensando la hispanidad. Estrategias para el estudio crítico de la historia del pensamiento filosófico-político hispánico”. Desafíos (Bogotá, Colombia) (15): 341-369. Fecha de consulta: 20 de febrero de 2015. https://revistas. urosario.edu.co/index.php/desafios/article/view/764

Unesco. 2005. La Educación de Jóvenes y Adultos en América Latina y el Caribe Hacia un Estado del Arte. Santiago de Chile. Fecha de consulta: 20 de febrero de 2015. http://unesdoc.unesco.org/images/0013/001389/138996s.pdf

Universidad Andrés Bello. 2005. "Memoria para optar al Título Profesional de Administrador en Ecoturismo". Santiago de Chile. Fecha de consulta: 20 de febrero de 2015. https://es.scribd.com/document/114954760/tesis-ecoturismo2005

Universidad Autónoma de Madrid, Departamento de Organización de Empresas. S. f. Proceso de seguimiento y evaluación cientifica de tesis doctorales. Fecha de consulta: 20 de febrero de 2015. https://www.uam.es/cs/BlobServer?blobkey=id\&blobwhere $=1242664262726$ \&blobheader $=$ application $\% 2 F p d f \& b l o-$ bheadername $2=$ pragma\&blobheadername $1=$ Contentdisposition\&blobheader value $2=$ public \&blobheadervalue $1=$ attachment $\% 3 B+$ filename $\% 3$ DDocumento+Seguimiento $+y+$ Evaluacion + Tesis + Doctorales. pdf\&blobcol=urldata\&blobtable $=$ MungoBlobs

Universidad de Antioquia. Dirección de Regionalización. S. f. El Estado del Arte. Fecha de consulta: 20 de febrero de 2015. https:/www.academia.edu/6767148/ UNIVERSIDAD_DE_ANTIOQUIA

Universidad de Granada. 2009. Animación digital y realidad aumentada. Fecha de consulta: 31 de mayo de 2009. http://www.ugr.es/ cm/cursos/a10.htm

Universidad de la Sabana. S. f. "El papel de la teoría en la investigación científica", conversatorio. Fecha de consulta: 14 de mayo de 2009. http://www.unisabana. edu.co/investigacion/docs/conversatorio.pdf 
Universidad de Málaga. 2008. Calendario de eventos, Jornada Universidad- Empresa, 17- 23 de abril de 2008. Bic Euronova, Centro Europeo de Empresas e Innovación de Málaga y Oficina de Transferencia de Resultados de Investigación OTRI. Fecha de consulta: 31 de mayo de 2009. http://www.reta.es/index.php/actualidad/ agenda.html?task=view_detail \&agid $=179 \&$ year $=2008 \&$ month $=04 \&$ day $=22$

Universidad de Málaga. 2006 (30 de noviembre). La Universidad de Málaga entra en el Programa Campus de InterSystems. Fecha de consulta: 20 de febrero de 2015. http://diarioti.com/la-universidad-de-malaga-entra-en-el-programa-campus-de-intersystems/12904

Universidad de Oviedo. 2002. "Publicaciones en revistas y en libros, Lógica y Filosofía de la Ciencia”, en Memoria 2002. Departamento de Filosofía. Fecha de consulta: 31 de mayo de 2009. http://www.uniovi.es/vicinves/Web_investigacion/ Memoria/mem2002/pdf/D4210.pdf

Universidad de Vigo. 2005. Grupo de Cultivo de Tejidos y Biotecnología de la EELM. Fecha de consulta: 31 de mayo de 2009. http://webs.uvigo.es/red.biotec.frutales/ Grupo\%20CSIC\%20La\%20Mayora.pdf

Universidad del Rosario. S. f. Presentación del Programa. Escuela de Ciencias Humanas, Maestría en Filosofía. Fecha de consulta: 31 de mayo de 2009. http://www. urosario.edu.co/cienciashumanas/downloads/maestria_filosofia.pdf

Universidad Pedagógica Nacional. 2009. Condiciones de posibilidad de la filosofía y su enseñanza, seminario-taller. Bogotá, 2-6 de febrero. Fecha de consulta: 31 de mayo de 2009. http://www.germanvargasguillen.com/evento\%20inaugural\%20licenciatura.pdf

Universidad Torcuato di Tella. Área Educación. S. f. Coordenadas en Investigación Educativa. Fecha de consulta: 14 de mayo de 2009. http://www.escuelamarinavilte.org.ar/_paginas/dhie/links/coordenadas_nv_duc.doc

Vargas, L. 2009. "Por qué nos oponemos a una didáctica basada en normas de competencia", documento presentado en el foro Arte en Filosofía. Fecha de consulta: 31 de mayo de 2009. http://www.camdf.sep.gob.mx/actualizArte/r0/filosofia/ Filosofia.pdf

Vélez O. L. y M. E. Galeano. 2002. Estado del arte sobre fuentes documentales en investigación cualitativa. Medellín: Universidad de Antioquia.

Woellner, L. et al., eds. 2002. Ciência, Tecnologia e Sociedade: O desafio da interação. Londrina, Brasil: IAPAR.

Para citar este texto:

Posada González, Nubia Leonor. 2017. "Algunas nociones y aplicaciones de la investigación documental denominada estado del arte". Investigación Bibliotecológica: archivonomía, bibliotecología e información 73 (31): 237 -263.

http://dx.doi.org/10.22201/24488321xe.2017.73.57855 